\title{
AN ANALYSIS OF RESOLIDIFICATION IN WELDING*
}

\author{
BY \\ GUNDUZ CAGINALP \\ University of Pittsburgh
}

I. Introduction. We consider a moving boundary problem which arises from welding. The problem may be described as a localized heat source moving at constant velocity on a sheet of metal. Hence, the liquid metal forms a small pool surrounded by the solid metal. Resolidification occurs at the tail end of the weld pool, thereby forming resolidification patterns which are of great practical importance in terms of the strength of the resulting bond. Experimentally, it is found that low velocities produce resolidification patterns which diverge logarithmically along the line followed by the source [1-8]. For sufficiently high velocities, however, the resolidification patterns intersect at a finite angle, resulting in a fault line which usually results in a weak bond.

One possible explanation for this phenomenon has been the formation of a sharp vertex as the tail end of the weld pool once the system is above some initial velocity [7]. In a previous paper [9] it has been proven that such a free boundary was not possible within the context of a reasonable two-dimensional model.

In this paper we consider an explanation based on the curvature of the end of the weld pool. The basic idea is to model the end of the weld pool as an infinite parabola and to assume isotherms are parallel to the parabola. This transforms the free boundary problem into an overdetermined ordinary differential equation and forces a relation between the velocity, $v$, and the radius of curvature, $a$, of the parabola. In particular, there is an inverse relationship between the velocity and radius of curvature. This result compares reasonably well with experiments performed on titanium [8].

The explanation of the fault line is then based on two physical length scales in this problem: the radius of curvature and the mean separation between the grain boundaries in the metal. If the radius of curvature is large in comparison with the distance between grain boundaries, then the resolidification occurs smoothly. If the two lengths are comparable, then the grain boundaries from the two directions will tend to collide and form a new grain boundary which is a jagged line along the welding arc line. This is known as the fault line. Hence there is a critical curvature (corresponding to a critical velocity) beyond which the fault line forms.

\footnotetext{
* Received March 29, 1985.
} 
The mathematical model we consider is a Stefan problem in two dimensions. We let $\Omega_{1}$, $\Omega_{2}$ denote the liquid and solid, respectively, while $\Gamma$ denotes the interface. We assume that the source of heat, i.e., the welding arc, is localized so that the source term vanishes near the tail end of the weld pool. In each phase, then, one has the heat equation,

$$
\partial u / \partial t=A \Delta u \quad\left(\text { in } \Omega_{1}, \Omega_{2}\right),
$$

where $u=T-T_{M}$ is the reduced temperature, with $T_{M}$ the melting temperature, and $A$ is the thermal diffusivity. Across the interface one has the latent heat equation

$$
l v_{n}=(K / \rho)\left(\nabla u_{S}-\nabla u_{L}\right) \cdot \hat{n} \quad(\text { on } \Gamma) \text {, }
$$

where $l$ is the latent heat of fusion (per unit mass), $v_{n}$ is the normal velocity of the interface, $K$ is the thermal conductivity, $\rho$ is the mass density, $\hat{n}$ is the unit normal vector into the liquid, and $\nabla u_{S}$ and $\nabla u_{I}$ are the gradients of the temperature at the interface from the solid and liquid directions, respectively.

In addition to Eq. (1.2) one must specify the temperature at the interface. A physically realistic condition is the Gibbs-Thompson relation [10-13]

$$
u=\sigma \kappa / \Delta S \quad(\text { on } \Gamma) \text {, }
$$

where $\sigma$ is the surface tension, $\kappa$ is the curvature, and $\Delta S$ is the difference in entropy in the two phases. For the type of experimental situation we consider, however, the curvature is sufficiently small that one may use the approximation

$$
u=0 \quad(\text { on } \Gamma) \text {. }
$$

The transformations we perform are also applicable to a condition of the form

$$
u=f(a) \quad(\text { on } \Gamma)
$$

where $a$ is the radius of curvature at the tip of the weld pool.

II. Basic transformations. Let the source (i.e., welding arc) move with constant speed $v$ in the positive $y$ direction. In the moving coordinates

$$
x=x^{\prime}, \quad y^{\prime}=y+v t, \quad t^{\prime}=t,
$$

a steady state solution to (1.1) must satisfy

$$
\Delta u-\frac{v}{A} \frac{\partial u}{\partial y^{\prime}}=0 .
$$

We now focus attention on the tail end of the weld pool and model this region with an infinite parabola. We may transform the equations (2.2) and (1.2) into parabolic coordinates using a transformation due to Ivantov [14]:

$$
\begin{gathered}
T:\left(x^{\prime}, y^{\prime}\right) \rightarrow(\tilde{x}, \tilde{y}), \\
\tilde{x}=x^{\prime}, \quad \tilde{y}=-y^{\prime}-\sqrt{x^{\prime 2}+y^{\prime 2}} .
\end{gathered}
$$

For any constant $a$, the equation $\tilde{y}=a$ is equivalent to

$$
y^{\prime}=\frac{x^{\prime 2}}{2 a}-\frac{a}{2} \text {. }
$$


The unit normal, $\hat{n}$, toward the inside of the parabola is given by

$$
\hat{n}=\left(-x^{\prime}, a\right) / \sqrt{x^{\prime 2}+a^{2}} \text {. }
$$

Upon transforming the heat diffusion and latent heat equations and considering solutions which depend only on $\tilde{y}$, we have the equations:

$$
\begin{gathered}
\frac{d^{2} u}{d \tilde{y}^{2}}+\frac{1}{2}\left[\frac{1}{\tilde{y}}+\frac{v}{A}\right] \frac{d u}{d \tilde{y}}=0 \quad(\tilde{y} \neq a), \\
-\frac{1}{2} l v=\frac{K}{\rho}\left[\left(\frac{d u^{S}}{d \tilde{y}}-\frac{d u^{L}}{d \tilde{y}}\right)\right]_{\tilde{y}=a} .
\end{gathered}
$$

Far from the interface, we assume the material is at a fixed temperature in each phase, i.e., $u(\tilde{y})$ satisfies

$$
\begin{aligned}
& u(-\infty)=u_{0}, \\
& u(+\infty)=u_{\infty} .
\end{aligned}
$$

At the interface, one has the condition

$$
u(a)=0 .
$$

Hence, we consider the problem of finding a solution to (2.6)-(2.10). This is an overdetermined system and therefore forces a relation between $v$ and $a$. If we let

$$
w \equiv d u / d \tilde{y},
$$

then (2.6) becomes

$$
\frac{d w}{d \tilde{y}}+\frac{1}{2}\left(\frac{1}{\tilde{y}}+\frac{v}{A}\right) w=0
$$

The solution is found to be

$$
|w|=C \frac{e^{-v \tilde{y} / 2 A}}{|\tilde{y}|^{1 / 2}} .
$$

Solving for $u$, one obtains

$$
\begin{aligned}
u(\tilde{y}) & =\bar{C}_{1} \int_{v b / 2 A}^{v \tilde{y} / 2 A} \frac{e^{-s}}{|s|^{1 / 2}} d s+\bar{C}_{2}(b) \\
& =C_{1} E_{C}\left[\left(\frac{v \tilde{y}}{2 A}\right)^{1 / 2}\right]+C_{2},
\end{aligned}
$$

where the complementary error function is defined by

$$
E_{C}(x)=\frac{2}{\sqrt{\pi}} \int_{x}^{\infty} e^{-s^{2}} d s .
$$

If we consider the boundary conditions imposed on the solid, i.e., (2.9) and (2.10), we find for $\tilde{y} \geqslant a$

$$
u(\tilde{y})=\frac{-u_{\infty}}{E_{C}\left[\left(\frac{r u}{2 A}\right)^{1 / 2}\right]} E_{C}\left[\left(\frac{v \tilde{y}}{2 A}\right)^{1 / 2}\right]+u_{\infty}
$$


Thus, the temperature of the solid is determined entirely from these boundary conditions. The latent heat equation (2.7) then implies an overdetermined system, thereby forcing a relationship between $v$ and $a$. At this point we make the physical assumption that the gradient of the temperature in the liquid is sufficiently small and may be neglected.

Applying the latent heat condition, (2.7), we have the equation

$$
-\frac{1}{2} l v=\frac{1}{2 \sqrt{\pi}} \frac{K}{\rho} \frac{v}{A} \frac{u_{\infty}}{E_{C}\left[\left(\frac{r a}{2 A}\right)^{1 / 2}\right]} e^{-v a / 2 A}\left(\frac{v a}{2 A}\right)^{-1 / 2} .
$$

Upon defining the two dimensionless constants

$$
\alpha \equiv\left(\frac{v a}{2 A}\right)^{1 / 2}, \quad \beta \equiv \frac{-u_{\infty} K}{\sqrt{\pi} l \rho A},
$$

one may write (2.17) as

$$
F(\alpha) \equiv \alpha e^{\alpha^{2}} E_{C}(\alpha)=\beta .
$$

Thus, equation (2.19) expresses the relationship between the dimensionless parameters involving $v$ and $a$. In particular, this equation asserts an inverse relationship between $v$ and $a$.

We note that the interfacial condition (2.10) could be replaced by the equation

$$
u(a)=f(a)=\sigma / a \Delta S
$$

since the curvature of the tip of the parabola is just $a$. This would be a better approximation to (1.3). The analog of Eq. (2.16) would then be

$$
u(\tilde{y})=\frac{-u_{\infty}+\sigma / a \Delta S}{E_{C}\left[\left(\frac{v a}{2 A}\right)^{1 / 2}\right]} E_{C}\left[\left(\frac{v \tilde{y}}{2 A}\right)^{1 / 2}\right]+u_{\infty} .
$$

With the introduction of the curvature term, the constraining Eq. (2.19) now differs in that $\beta$ must be redefined as

$$
\bar{\beta} \equiv \frac{\left(-u_{\infty}+\sigma / a \Delta S\right) K}{\operatorname{l\rho } A} .
$$

This means that $v$ and $a$ are no longer reciprocals of one another. However, if the surface tension $\sigma$ is small, then one can presumably treat the curvature term $\sigma a / \Delta S$ as a perturbative term in the equation $F(\alpha)=\beta$. For larger values of $\sigma$ one may still obtain a relation between $v$ and $a$ which may be compared with experiments.

III. Application to fault line. We begin with Eq. (2.19), which represents a one-to-one relationship between $v$ and $a$. As $v$ is increased the radius of curvature of the tip of the parabola, i.e., $a$, is decreased. At some critical velocity, $v_{c}$, which corresponds to a critical radius of curvature, $a_{c}$ in our analysis, the resolidification pattern of the grain boundaries ceases to be a smooth set of curves along the welding arc, and forms what is known as the fault line. This may be explained by the ansatz that this critical radius of curvature is an $\mathcal{O}(1)$ multiple of the distance between grain boundaries of the metal.

To see why the fault line occurs at this curvature and velocity, we present a brief discussion of the behavior of the grain boundaries [15]. The question of the strength of the welding bond and the occurrence of a fault line is closely related to the nature of the 
collisions or coalescence between grain boundaries. Two of the main reasons for collisions are the following:

(1) The intersection between the grain boundaries and the solid-liquid interface will not exactly be perpendicular so that eventually the grain boundaries will intersect as shown in Fig. 1. For slow speeds of welding, this will be the main mechanism for collisions between grain boundaries. These collisions will result in new grain boundaries which will have some general direction but not one which is very coherent.

(2) Another mechanism is due to large curvature, which the grain boundaries attempt to reduce by migration. The solid line in Fig. 2 shows the original shape of the grain boundary, while the dotted line shows the relocation which lowers the curvature. Since the

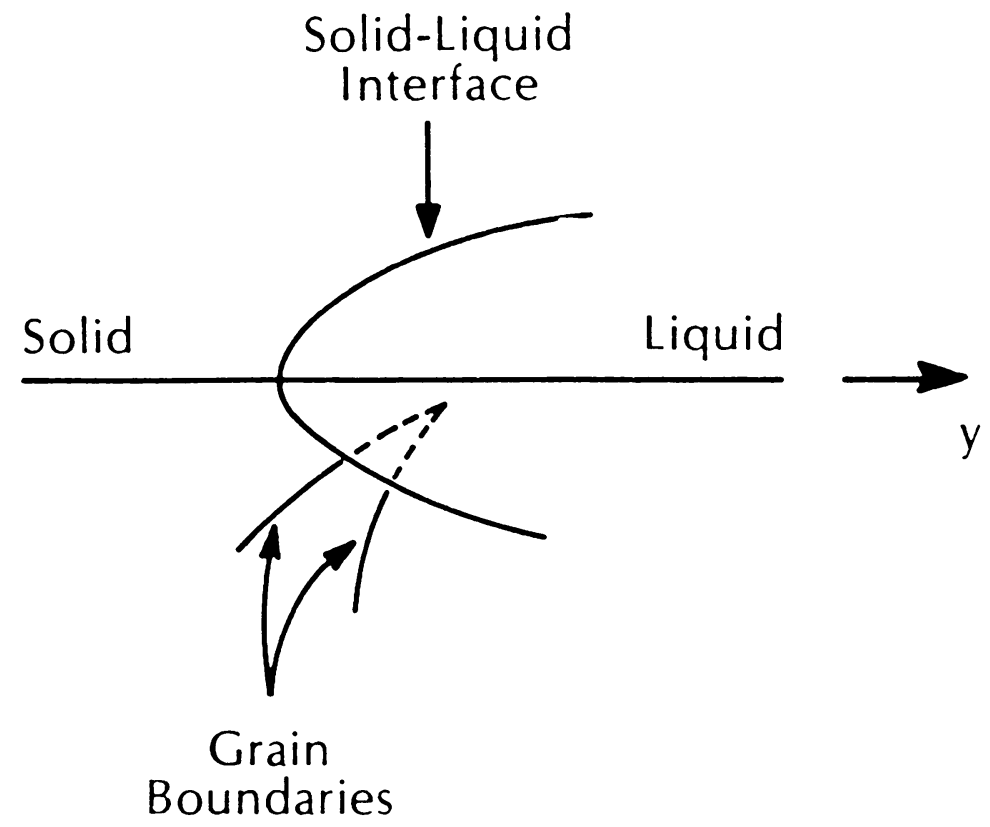

FIG. 1
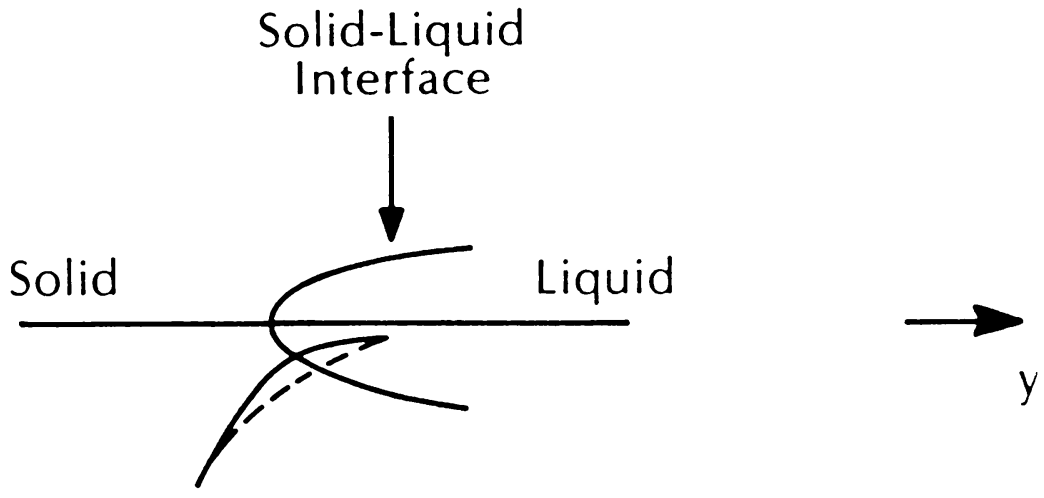

FIG. 2 
ones closer to the tip migrate further, one can expect collisions. After a collision, as shown in Fig. 3(a), equilibration of the angle of intersection results in a reconfiguration of the grain boundaries (see Figure 3(b)). The new grain boundaries formed will have a more coherent direction than those resulting from (1).

If we consider the grain boundary formed near the locus of the welding arc, then we may consider the angle $\theta$ between this boundary and the $y$-axis (see Fig. 4). Hence, we may define a measure of the directionality of these grain boundaries along the $y$-axis by a functional such as

$$
S[\theta]=\int_{A}^{B}|\cos \theta(y)| d y .
$$

IV. Comparison with experiment. In this section we compare the implications of the ideas discussed in Secs. II and III with experiments performed by L. Brush [8] on titanium. In these experiments, a welding arc was used on a sheet of titanium at various different velocities. The metal was photographed during and after the experiments to
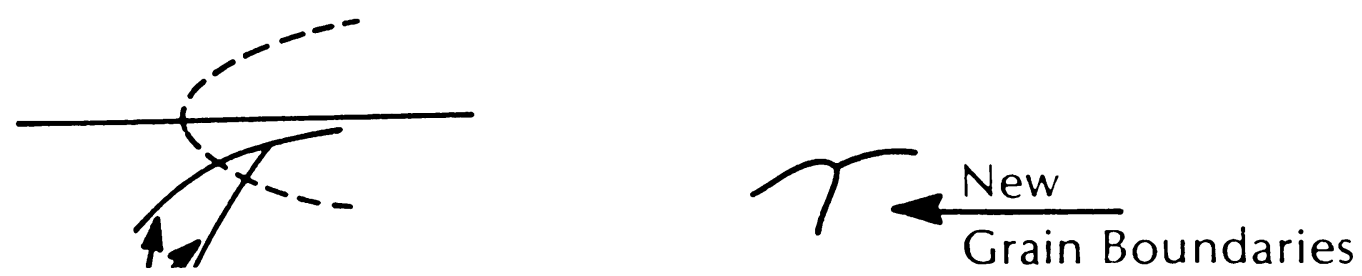

\section{Grain \\ Boundaries}

(a)

(b)

Fig. 3

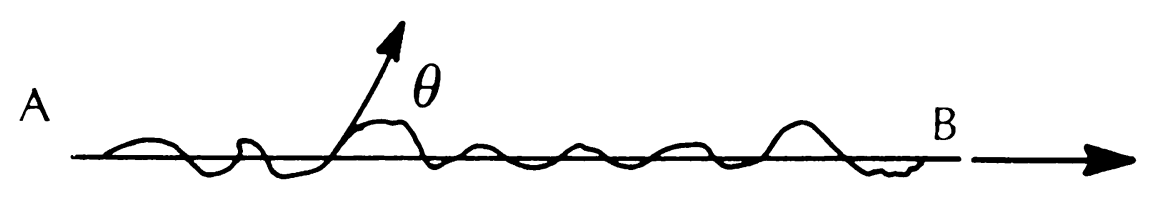


indicate the size and shape of the weld pool as well as the resulting resolidification patterns.

The analysis of Sect. III appears to be justified since the fault line seems to occur at a velocity (approximately $25 \mathrm{~cm} / \mathrm{min}$ ) which corresponds to a radius of curvature of the tip of the weld pool which is approximately that of the distance between the grain boundaries. See Photos 1, 2, 3, 4 .

A more detailed comparison may be performed for the implications of Sec. 2. In particular, one would like to check whether the relation

$$
v=C a^{-1}
$$

is satisfied for a particular constant $C$ and whether this constant $C$ then implies that the parameter $u_{\infty}$ (the effective temperature at a distance which is large in comparison with the radius of curvature of the parabola) is a reasonable value.

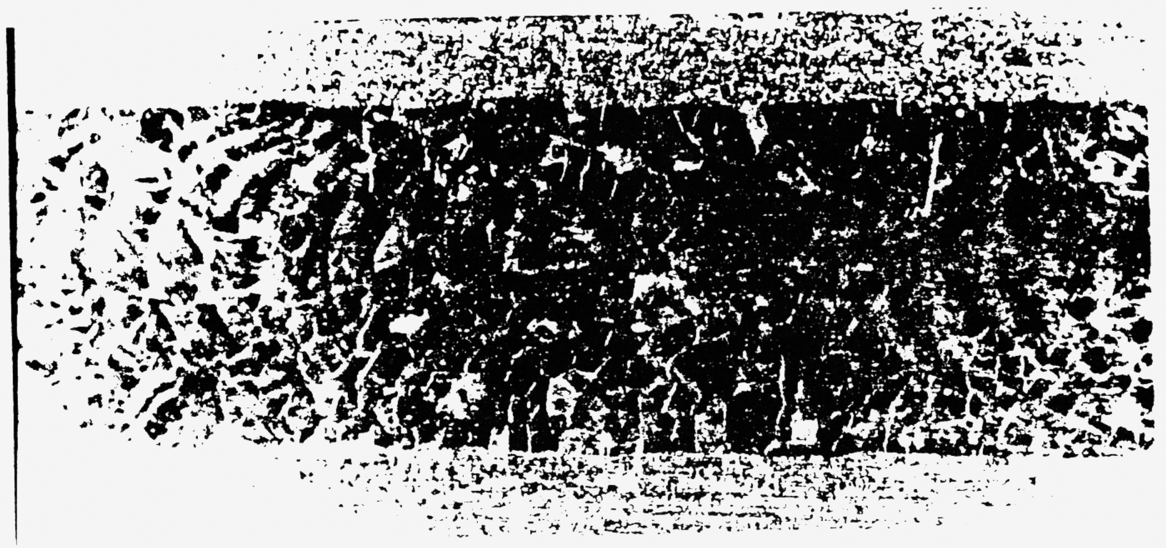

Рното 1. A sheet of titanium after a pulsed welding arc has passed at 4 inches per minute. The pulsing freezes the melt quickly so that the shape of the weld pool is apparent. All experiments and photos produced by L. Brush.

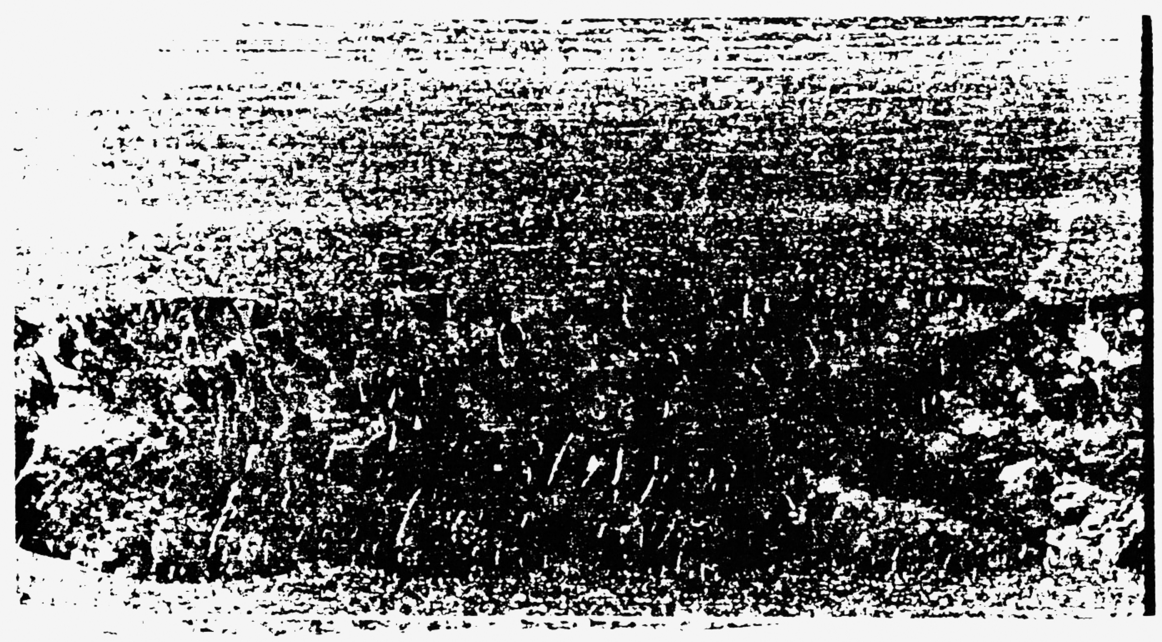

Рното 2. A sheet of titanium after a pulsed welding arc has passed at 14 inches per minute. 


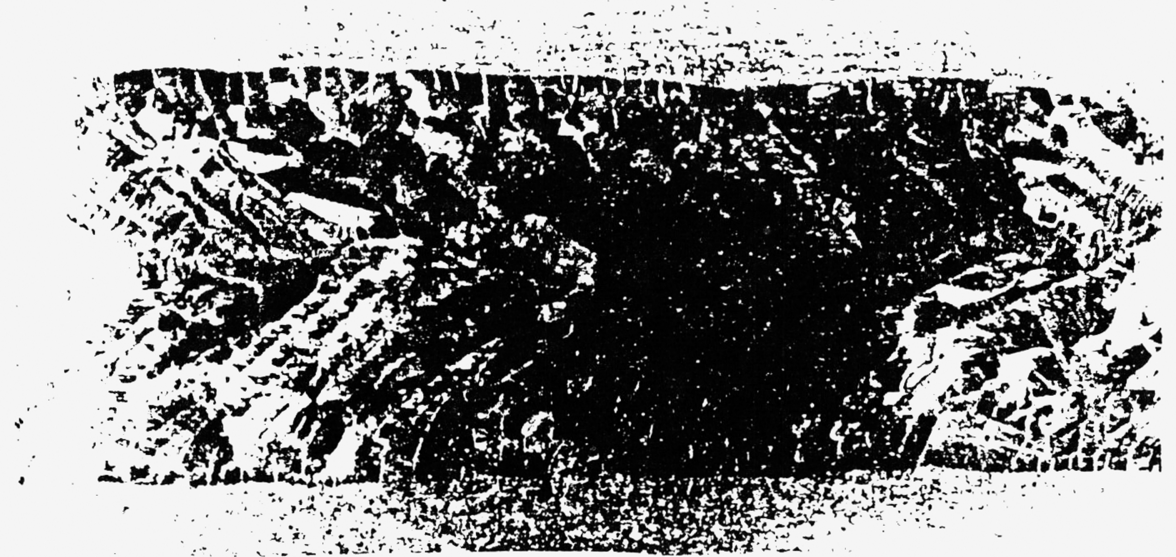

Рното 3. A sheet of titanium after a welding arc has passed at a constant rate of 6 inches per minute. This is sufficiently slow so that a fault line does not form, i.e., there is no distinct line forming a path of intersections of grain boundaries.

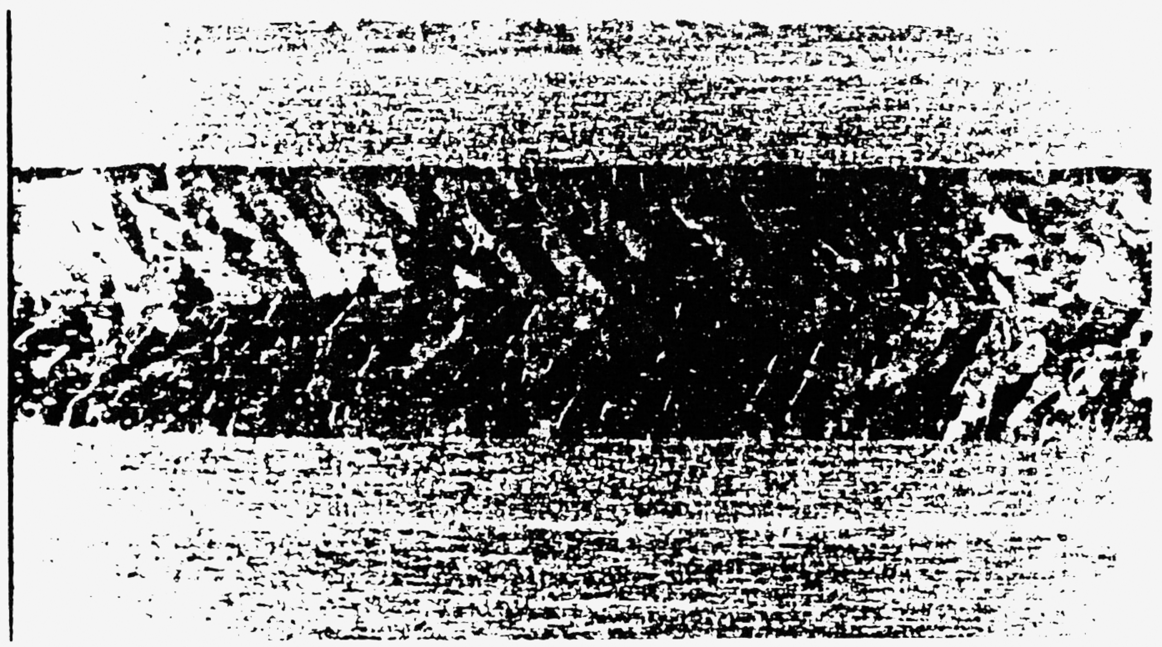

Рното 4. A sheet of titanium after a welding arc has passed at a constant rate of 18 inches per minute. This is above the velocity necessary to form a fault line. There is a distinct, almost straight line formed by the intersections of the grain boundaries.

The values of the basic constants for titanium are given in [16] as the following:

$$
\begin{aligned}
l & =100 \mathrm{cal} / \mathrm{g}, \\
\rho & =4.15 \mathrm{~g} / \mathrm{cm}^{3}, \\
A & =0.59 \mathrm{~cm}^{2} / \mathrm{sec}, \\
K & =.46 \mathrm{cal} / \mathrm{sec}-\mathrm{cm}-{ }^{\circ} \mathrm{C} .
\end{aligned}
$$

The dimensionless constant $\beta$ is then given by

$$
\beta=\frac{-0.0019}{\sqrt{\pi}} \frac{u_{\infty}}{{ }^{\circ} \mathrm{C}} .
$$


The experiments for titanium [8] indicate that

$$
v a \cong 0.051 \mathrm{~cm}^{2} / \mathrm{sec} \quad \text { (experimental) }
$$

(see Photo 1) and the parameter $\alpha$ is then given by

$$
\alpha \cong 0.207 \text {. }
$$

Equation (2.19) then implies

$$
0.166=F(0.207)=-0.0011 u_{\infty} /{ }^{\circ} \mathrm{C}
$$

so that the effective temperature is given by

$$
u_{\infty}=-157^{\circ} \mathrm{C} \text {. }
$$

This implies that the temperature is $157^{\circ} \mathrm{C}$ below the freezing temperature when the distance from the top of the parabola is such that the parabolic isotherms cease to be a good approximation. This distance is presumably a small multiple of the radius of curvature. Hence, the calculation (4.7) is physically reasonable.

Finally, we compare Eq. (4.1) with experiment. The constant value, C, in (4.1) which corresponds to (4.7) is 0.05 . The curve

$$
a=0.05 / v
$$

is plotted in Fig. 5, along with the experimental values. The agreement between the reciprocal relationship and the experimental results seems to be quite good. One must be cautioned, however, that the radius of curvature has been obtained by measurements on the photographs using a compass (by the author) and is therefore subject to a reasonable amount of error.

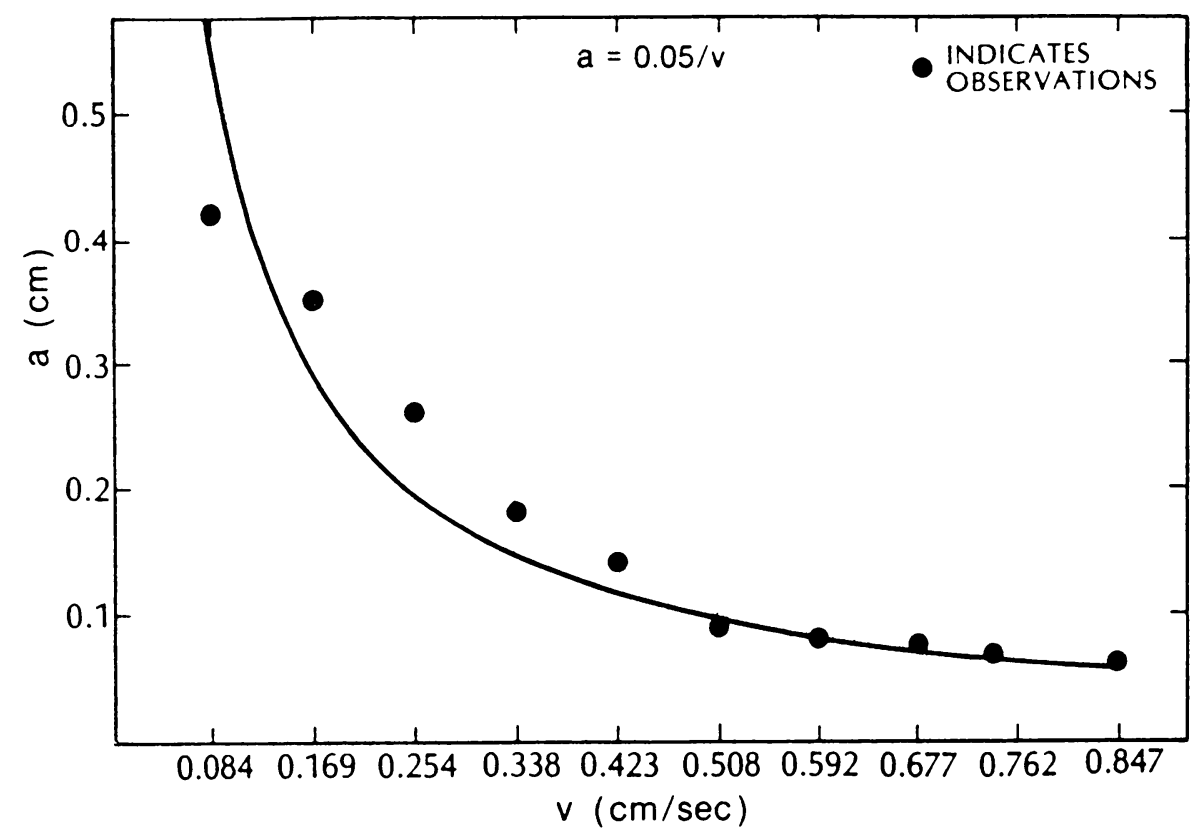


The analysis and comparisons presented in this paper suggest several experiments. A series of experiments performed on sheets of the same metal with different spacing between grain boundaries would further test the hypothesis involving the relationship between this spacing and the curvature at which the fault line occurs. Similar experiments on different metals would further test the accuracy of the inverse relation between $v$ and $a$.

Acknowledgments. The author is greatly indebted to Professor W.W. Mullins for discussions leading to the explanation of the fault line in Sec. III. Thanks are also due to L. Brush and Professor R. Sekerka for access to experimental results and numerous discussions. The support of the National Science Foundation through Grant No. DMS8403184 is gratefully acknowledged.

\section{REFERENCES}

[1] H. Nakagawa, M. Kato, F. Matsuda, T. Senda, X-ray investigations on solidification structures in weld metal, Trans. JWS 1, 94-103 (1970)

[2] __ Crystallographic anisotropy of columnar crystal zone in aluminum weld metal, Trans. JWS 2, 10-20 (1970)

[3] _ Crystallographic investigation of origination of new columnar crystal sheet, Trans. JWS 2, 1-9 (1971)

[4] _ Fundamental solidification mechanism and microstructures in GTA spot welds of aluminum sheets, Trans. JWS 1, 28-39 (1970)

[5] _ Features in weld solidification structure of high purity aluminum sheet, Trans. JWS 4, 14-19 (1973)

[6] W. F. Savage, C. D. Lundin, and A. H. Aronson, Weld metal solidification mechanics, Weld J. 44, 175-181 (1965)

[7] W. F. Savage, Solidification, segregation and weld imperfections, 1980 Houdremont Lecture Welding in the World 18, 89-113 (1980)

[8] L. Brush, Weld pool transitions in autogenous full penetration welds, Master's Thesis, Carnegie-Mellon University (1983)

[9] G. Caginalp, A free boundary problem with moving source, Adv. in Appl. Math. 5, 476-488 (1984)

[10] J. W. Hoffman and J. W. Cahn, A vector thermodynamics for anisotropic surfaces, I: Fundamentals and application to plane surface junctions, Surf. Sci. 31, 368-388 (1972)

[11] J. W. Cahn and D. W. Hoffman, A vector thermodynamics for anisotropic surfaces. II: Curved and faceted surfaces, Acta Metallurgica 22, 1205-1214 (1974)

[12] W. W. Mullins, The thermodynamics of crystal phases with curved interfaces: Special case of interface isotropy. and hydrostatic pressure, Proc. Internat. Conf. on Solid-Solid Phase Transformations, H. I. Erinson, et al., eds., TMS-AIME, Warrendale, PA (1983)

[13] W. W. Mullins, Thermodynamic equilibrium of a crystal sphere in a fluid, Carnegie-Mellon University research report (1983)

[14] G. P. Ivantov, The temperature field around a spherical, cylindrical or pointed crystal growing in a cooling solution, Dokl. Akad. Nauk SSSR 58, 567-569 (1947)

[15] W. W. Mullins, Private communication

[16] Handbook of chemistry and physics, R. C. Weast, ed. Chemical Rubber Publishing Co. 55th ed., 1974, page D-152. 